SELECTED PAPER AT NCSP'14

\title{
Polygonal Structure Models of Ring Inhibitory Neural Network
}

\author{
Yuki Shoji, Yoko Uwate and Yoshifumi Nishio \\ Tokushima University \\ 2-1 Minami-Josanjima, Tokushima 770-8506, Japan \\ Phone: +81-88-656-7470, FAX: +81-88-656-7471 \\ E-mail: $\{$ shoji, uwate, nishio\} @ee.tokushima-u.ac.jp
}

\begin{abstract}
The ring inhibitory neural network is a typical neural system used to generate oscillation with a simple structure. Neurons in the ring inhibitory neural network are connected in one direction. In this study, we propose polygonal structure models of the ring inhibitory neural network that are composed of multiple ring inhibitory neural networks. We confirm that the polygonal structure models based on triquetrous and pentagonal models composed of three and five neurons respectively can oscillate. In our simulations, the polygonal structure models show various synchronization and oscillation phenomena based on their network topology.
\end{abstract}

\section{Introduction}

The synchronization phenomena of coupled oscillators have been researched widely in the fields of communication systems, robot engineering, biological systems, social networks, and so forth, mainly using the van der Pol oscillator and the Kuramoto model. On the other hand, the brain network and other biological neural networks can also cause synchronization. The oscillation and synchronization phenomena of neural networks arise from neurons. However, coupled oscillator models based on neurons have not been investigated sufficiently. It is important to investigate oscillator models based on neurons in order to reveal various complex synchronization phenomena in the brain.

Artificial neural networks are calculation models that can replicate some functions of the human brain. In particular, a neural network with oscillation is applied to pattern recognition and walking rhythm outbreak circuits [1]-[4]. A ring inhibitory neural network is a typical oscillation model with a simple structure and it is composed of neurons connected in one direction. The weights between the neurons are defined as negative because the neurons composing the system are inhibitory [5].

In this study, we focus on large-scale networks based on the ring inhibitory neural network as the neural oscillator. We propose polygonal structure models of the ring inhibitory neural network and investigate their synchronization and oscillation phenomena.

\section{Oscillation Systems 2.1 Ring inhibitory neural network}

Neural networks with oscillation are classified into two types: networks with self-connection and without selfconnection such as ring inhibitory neural networks. The ring inhibitory neural network is composed by connecting neurons in one direction. Figure 1 shows a general model of the ring inhibitory neural network. The dynamical system of this model is described by Eqs. (1) and (2). Moreover, the output function is defined as the arctangent.

$$
\begin{gathered}
\tau \frac{d u_{i}}{d t}=-u_{i}+W_{j i} f\left(u_{j}\right) \\
f(u)=\tan ^{-1} u
\end{gathered}
$$

Here $u$ is the internal value of each neuron and $\tau$ is the time constant. Neurons in the ring inhibitory neural network are connected unidirectionally with the negative weight $W_{j i}$. The oscillation phase of this system is the same as the number of neurons $m$. Oscillation is caused if the weight $W_{j i}$ is set to a negative value. In addition, this model can oscillate if the number of neurons is odd. We explain this condition by focusing on the equilibrium point. $u^{e}$ which has to satisfy Eq. (3). The state of $N_{1}$ is substituted sequentially in accordance with Eq. (4), when the number of neurons is $m$.

$$
\begin{gathered}
-u_{i}+W_{j i} f\left(u_{j}\right)=0 \\
u_{1}=W_{m 1} f\left(W_{m m-1} f\left(W_{m-1 m-2} \cdots W_{21} f\left(u_{1}\right) \cdots\right)\right)
\end{gathered}
$$

Equation (4) is a monotonically decreasing function if the number of neurons $m$ is odd. When the function $f$ is monotonically decreasing, the system has only one equilibrium point.

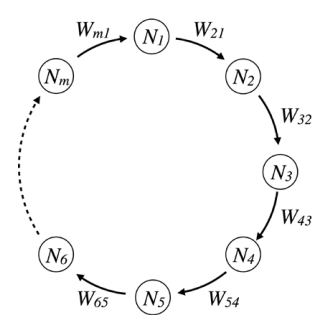

Figure 1: Ring inhibitory neural network 
Figure 2 shows the dynamics of the ring inhibitory neural network comprising three neurons. This model generates three-phase waveform. From Eqs. (1) and (2), the equilibrium point $u^{e}$ of the ring inhibitory neural network is the origin. In addition, the stability of the equilibrium point depends on the weight $W_{j i}$. We define the Jacobian matrix of the right side of Eq. (1) as matrix $A$. An eigenvalue $\lambda$ of matrix $A$ at the equilibrium point is calculated as follows.

$$
\operatorname{det}|A-\lambda E|=0
$$

The equilibrium point is unstable when the ring inhibitory neural network shows a limit cycle. This point is stable if the real parts of all eigenvalues are negative. Therefore, the ring inhibitory neural network form $=3$ shows a limit cycle if the weight is set to $W_{j i}<-2.0$.

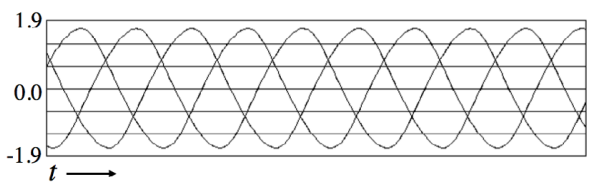

Figure 2: Dynamics of triquetrous ring inhibitory neural network ( $m=3, W_{j i}=-3.0, \tau=1.0$ )

\subsection{Polygonal structure models}

In this section, we explain the polygonal structure models. The conventional model of the ring inhibitory neural network can generate only an $m$-phase waveform if the number of neurons is $m$. We propose a ring inhibitory neural network with polygonal structures. The polygonal models are composed by connecting multiple conventional models under a certain condition. The neurons in this system stimulate each other complicated and show various oscillation phenomena. We can confirm the synchronization of neurons by investigating a polygonal structure. Moreover, the oscillation and synchronization are affected by the network topology. The dynamical system of this network is described by Eq. (6). The weights $W_{j i}$ between coupled neurons are all negative, whereas the weight $W_{j i}$ between disconnected neurons is defined as zero.

$$
\tau \frac{d u_{i}}{d t}=-u_{i}+\sum_{j=1}^{m} W_{j i} f\left(u_{j}\right)
$$

Moreover, we define some connection condition. The connections of neurons are set to one direction, which does not disturb circulation. This connection method follows a base model such as a conventional triquetrous or pentagonal model. In other words, the output of the neurons circulates through three neurons, for example, $N_{1}, N_{2}$, and $N_{4}$, as shown in Fig. 3(a). The network models of polygonal structure based on triquetrous and pentagonal models are shown in Figs. 4 and 5, respectively. The connection methods of the symmetric and asymmetric model with $m=10$ in
Figs. 5(a) and 5(b) include triquetrous and pentagonal structures. In this study, we classify these models into pentagonal models, because they generate five-phase synchronization.

In addition, polygonal structure models can be classified into two kinds, symmetric and asymmetric models, by the numbers of inputs and outputs. The polygonal models are classified as symmetric if the numbers of inputs and outputs of each neuron are equal as in Fig. 3(a). On the other hand, an asymmetric model does not satisfy this condition such as the model in Fig. 3(b).

Next, we focus on the stability and equilibrium point of the two polygonal models based on the triquetrous model. It is important that we analyze the equilibrium point to understand the characteristic behavior of the system. From Eqs. (2) and (6), the equilibrium point $u^{e}$ of each system is the origin. Each model can oscillate if each neuron generates a limit cycle around the origin. Therefore, the equilibrium point must be unstable. The eigenvalue $\lambda$ can be found from Eq. (5) if the Jacobian matrix of the right side of Eq. (6) is the matrix $A$. The oscillation condition of the asymmetric polygonal model for $m=7$ is $W_{j i}<-1.1$. Similarly, the symmetric polygonal model for $m=6$ is unstable when $W_{j i}<-1.0$.

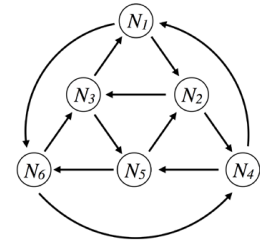

(a) Symmetric model

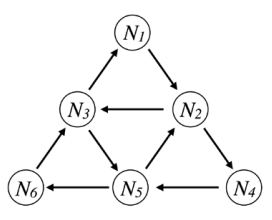

(b) Asymmetric model
Figure 3: Polygonal models for $m=6$

\section{Simulation Results}

In our simulation, we investigate the dynamics of polygonal structure models such as those Figs. 4 and 5 by the RungeKutta method. The weight $W_{j i}$ is set to $W_{j i}=-3.0$. The time constant $\tau$ is set to $\tau=2.0$.

\subsection{Polygonal models based on $m=\mathbf{3}$}

In this section, we give the simulation results for the polygonal structure models based on the conventional model composed of three neurons.

The polygonal model for $m=6$ in Fig. 4(a) is classified as a symmetric model. The oscillation of this polygonal model is shown in Fig. 6(a). This model for $m=6$ has a symmetric structure. It generates a three-phase waveform, and each wave pattern has the same amplitude. In contrast to the asymmetric model, the neurons at symmetric positions cause complete synchronization such as states $u_{1}$ and $u_{5}$. This complete synchronization is by solving the circuit equation.

By contrast, the neurons in the asymmetric model for $m=$ 6 in Fig. 4(b) cause different oscillation phenomena. The os- 


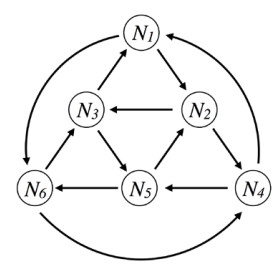

(a) Symmetric model for $m=6$

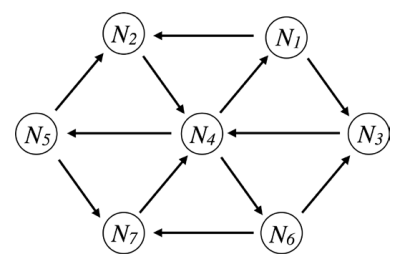

(c) Asymmetric model for $m=7$

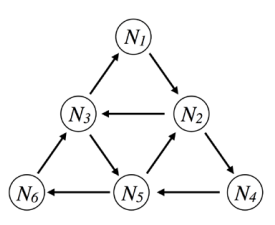

(b) Asymmetric model for $m=6$

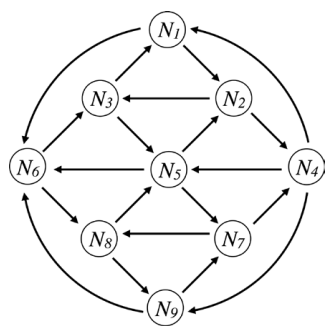

(d) Asymmetric model for $m=9$
Figure 4: Polygonal models based on $m=3$ (triquetrous model)

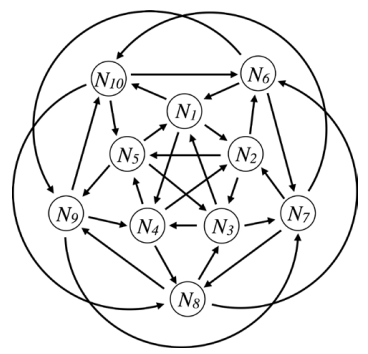

(a) Symmetric models for $m=10$
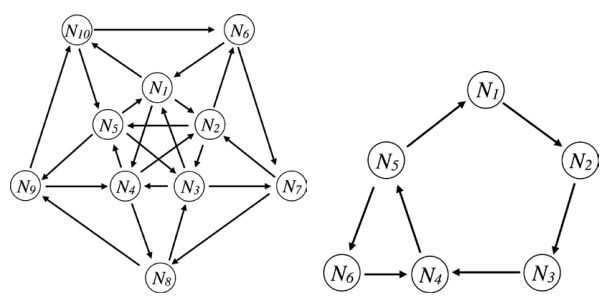

(b) Asymmetric models for (c) Asymmetric models $m=10$

for $m=6$

Figure 5: Polygonal models based on $m=5$ (pentagonal model)

cillation phenomena of the asymmetric model for $m=6$ are shown in Figs. 6(b) and 6(c). This asymmetric model for $m=6$ generates two kinds of three-phase waveform. These three-phase waveforms are generated by neurons with the same numbers of inputs and outputs. Specifically, neurons $N_{1}, N_{4}$ and $N_{6}$, which have one input and output generate a three-phase waveform. Moreover, the three-phase waveform of neurons $N_{2}, N_{3}$ and $N_{5}$ is the same as the oscillation of the symmetric model for $m=6$.

Figure 6(d) shows the oscillation of the asymmetric model for $m=7$ in Fig. 4(c). The asymmetric model for $m=7$ generates three wave patterns, and each pattern has a different amplitude. Neurons with the same numbers of inputs and outputs cause complete synchronization such as $u_{1}, u_{5}$ and $u_{6}$. This becomes clear by solving the circuit equation.

The polygonal model for $m=9$ in Fig. 4(d) is also classified as an asymmetric model. Figure 6(e) shows the oscillation of this model. This model generates nine wave pattern, and each wave pattern has a different amplitude. This model does not cause complete synchronization between each neuron, in contrast to the asymmetric model for $m=7$.

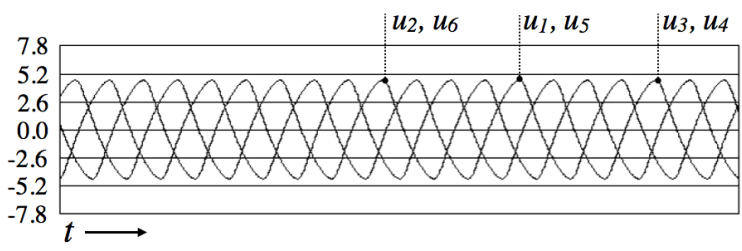

(a) Oscillation of symmetric model for $m=6$

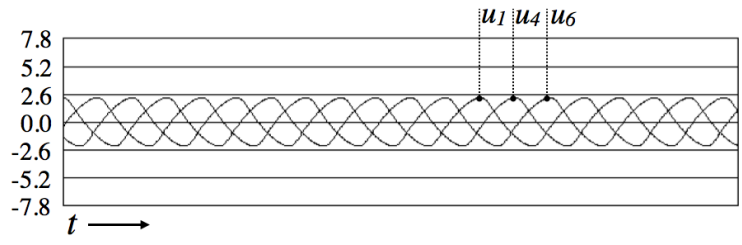

(b) Oscillation of asymmetric model for $m=6\left(N_{1}, N_{4}\right.$ and $\left.N_{6}\right)$

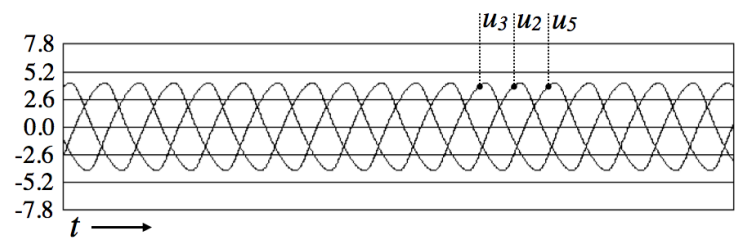

(c) Oscillation of asymmetric model for $m=6\left(N_{2}, N_{3}\right.$ and $\left.N_{5}\right)$

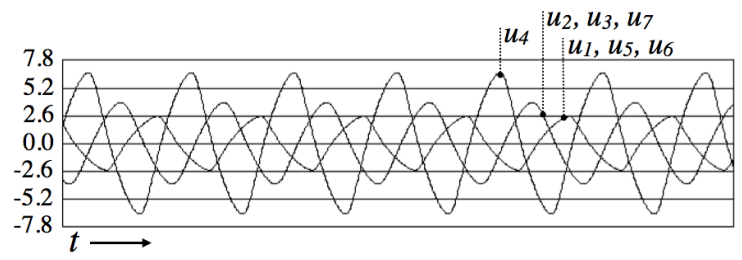

(d) Oscillation of asymmetric model for $m=7$

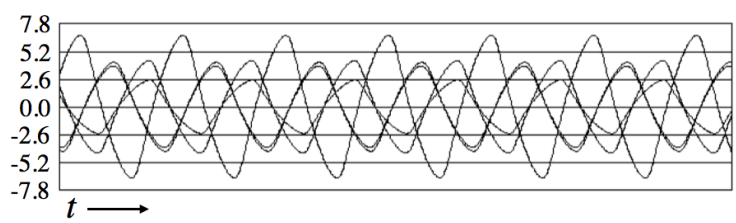

(e) Oscillation of asymmetric model for $m=9$

Figure 6: Oscillation of the polygonal models based on the triquetrous network for $m=3\left(W_{j i}=-3.0, \tau=2.0\right)$ 


\subsection{Polygonal models based on $\mathbf{m}=5$}

In this section, we give the simulation results for the polygonal models based on $m=5$ (pentagonal model). The conventional model for $m=5$ generates a five-phase waveform, and the neurons of this model have the same amplitude.

The model in Fig. 5(a) is the symmetric model for $m=10$. This model is symmetric, because the numbers of the inputs and outputs of all the neurons are the same. The oscillation of this model is shown in Fig. 7(a). The model for $m=10$ generates a five-phase waveform, and each wave pattern has the same amplitude, similarly to the symmetric model in Fig. 4(a). In addition, the neurons located at symmetrical position generate the same wave such as neurons $N_{1}$ and $N_{8}$. These models have the same characteristic as the polygonal models based on model for $m=3$.

Figure 5(b) shows the asymmetric model for $m=10$. The oscillation of this model is shown in Figs. 7(b) and 7(c). Two kinds of five-phase wave are generated simultaneously. The input and output values of neurons $N_{1}$ to $N_{5}$ are both three and those of neurons $N_{6}$ to $N_{10}$ are both two. Moreover, the five-phase waveform of $N_{6}$ to $N_{10}$ is the same as the oscillation of the symmetric model for $m=10$. These characteristics are also the same as those of the polygonal models based on the network model for $m=3$.

The network model in Fig. 5(c) is a combination of the triquetrous and pentagonal models. This model is asymmetric and generates the same oscillation phenomenon as the asymmetric model for $m=7$ in Fig. 4(c). Figure 7(d) shows the oscillation of this model. This model generates five-pattern oscillations and causes phase-synchronization between neurons. Moreover, neurons with the same numbers of inputs and outputs synchronize completely such as states $u_{1}$ and $u_{6}$. The amplitude of neuron $N_{4}$ is larger than the other neurons. A similar phenomenon was confirmed for the triquetrous asymmetric model for $m=7$. The neurons in an asymmetric model with more inputs oscillate with a larger amplitude.

\section{Conclusion}

In this study, we focused on the large-scale networks based on the neural oscillator and proposed polygonal structure models of the ring inhibitory neural network. These models cause synchronization which cannot be confirmed using coupled van der Pol oscillators. In addition, the polygonal structure models can be split into three categories and generate different waveforms in each model. Multi phase oscillation was confirmed in some polygonal models. In future, we will investigate general rules for the synchronization of each neuron and the oscillation conditions in detail.

\section{References}

[1] D. Wang: Emergent synchrony in locally coupled neural oscillators, IEEE Transactions on Neural Networks, Vols.

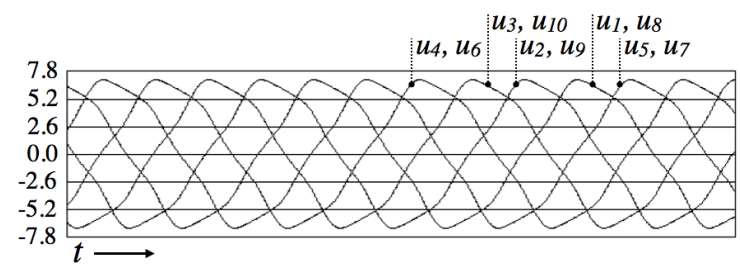

(a) Oscillation of symmetric model for $m=10$

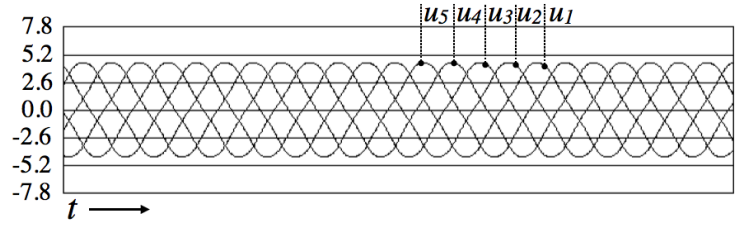

(b) Oscillation of asymmetric model for $m=10\left(N_{1}, N_{2}, N_{3}\right.$, $N_{4}$ and $N_{5}$ )

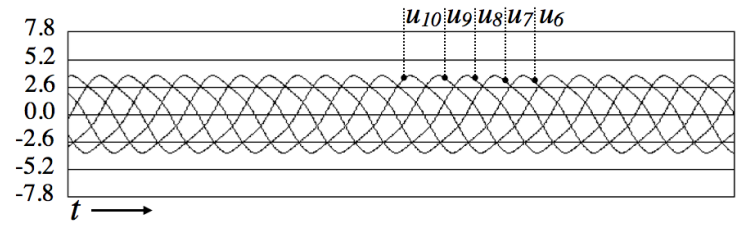

(c) Oscillation of asymmetric model for $m=10\left(N_{6}, N_{7}, N_{8}\right.$, $N_{9}$ and $N_{10}$ )

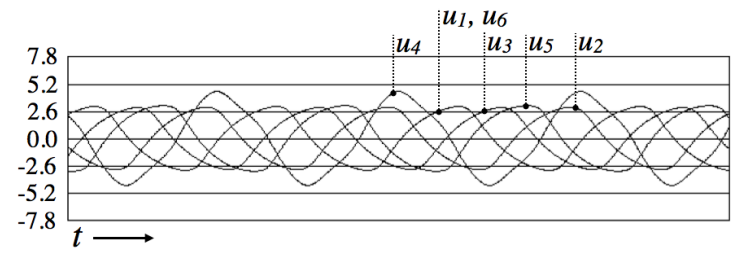

(d) Oscillation of asymmetric model for $m=6$

Figure 7: Oscillation of the polygonal models based on the pentagonal network for $m=5\left(W_{j i}=-3.0, \tau=2.0\right)$

6, No. 4, pp. 941-948, 1995.

[2] S. Still and G. L. Masson: Traveling waves in a ring of three inhibitory coupled model neurons, Neurocomputing, Vols. 26-27, pp. 533-539, 1999.

[3] G. B. Ermentrout and C. C. Chow: Modeling neural oscillations, physiology and behavior, Vols. 4-5, No. 77, pp. 629-633, 2002.

[4] G. Endo, J. Morimoto, J. Nakanishi and G. Cheng: An empirical exploration of a neural oscillator for biped locomotion control, Robotics and Automation, Proceedings of ICRA, Vol. 3, pp. 3036-3042, 2004.

[5] Y. Nakamura, Y. Nakano and H. Kawakami: N-phase oscillation of a neural oscillator connected with twisted ring structure, Electronics and Communications in Japan, Part 3, Vol. 83, No. 9, pp. 34-45, 2000. 